\title{
Combined Brillouin and Raman system for biomedical applications
}

\author{
Jan Rix ${ }^{1, *}$, Edmund Koch $^{1}$, Matthias Kirsch ${ }^{2,3,4}$, Gabriele Schackert ${ }^{3}$, Ortrud Uckermann $^{3}$, and Roberta Galli ${ }^{1,4}$ \\ ${ }^{1}$ Clinical Sensoring and Monitoring, Department of Anesthesiology and Intensive Care Medicine, Faculty of Medicine Carl Gustav \\ Carus, TU Dresden, Fetscherstrasse 74, D-01307 Dresden, Germany \\ ${ }^{2}$ Asklepios Kliniken Schildautal, Karl-Herold-Strasse 1, D-38723 Seesen, Germany \\ ${ }^{3}$ Neurosurgery, Faculty of Medicine Carl Gustav Carus, TU Dresden, Fetscherstrasse 74, D-01307 Dresden, Germany \\ ${ }^{4}$ National Center for Tumor Diseases (NCT), Partner Site Dresden, Fetscherstrasse 74, D-01307 Dresden, Germany
}

\begin{abstract}
A VIPA based Brillouin spectrometer consisting of commercially available components has been combined with a standard Raman spectrometer and a confocal microscope allowing simultaneous and colocalized measurements of biological samples and tissues.
\end{abstract}

\section{Introduction}

In the biomedical field, information about the stiffness of a tissue is of great importance as several cellular processes and mechanisms are depending on biomechanics. Especially in oncology, tumor tissue possesses a different stiffness compared to normal tissue [1], which might have an effect on cancer spreading and metastasis. Brillouin spectroscopy enables to analyze the mechanics of biological tissues in a non-destructive, label and contactfree manner. Furthermore, it can be combined with Raman spectroscopy, which enables addressing the compositional fingerprint of cells and tissue. The combined investigation of the biomechanical information obtained by Brillouin spectroscopy and the biochemical information by Raman spectroscopy provides a unique possibility of understanding complex biological mechanisms, as the molecular origin of changes in tissue's stiffness can be examined.

Therefore, a combined system for simultaneous acquisition of Raman and Brillouin spectra was built inside the research laboratory at the University Hospital of Dresden. First simultaneously acquired Brillouin and Raman spectra of different biomedical tissues were acquired in order to demonstrate the general practicability of combined measurements. Nearly transparent samples as well as opaque tissue samples were successfully measured.

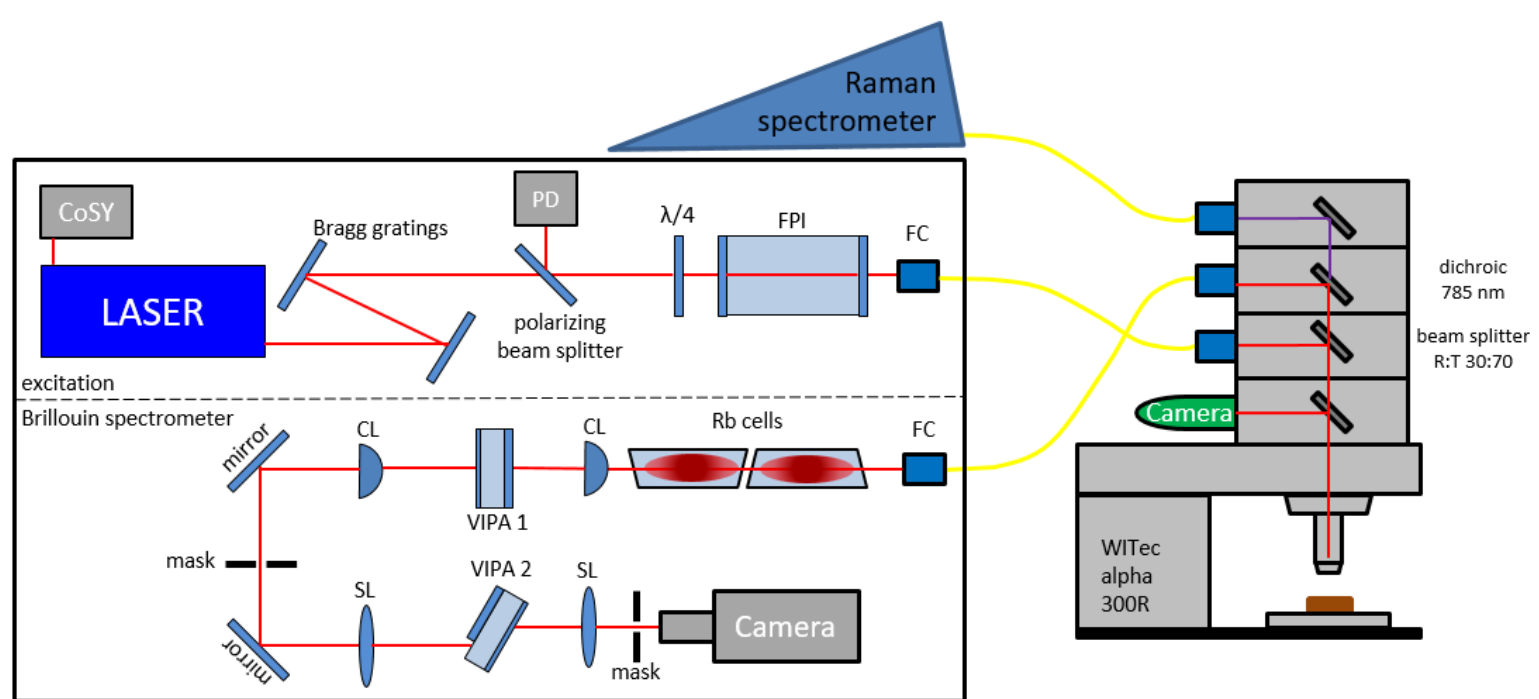

Fig. 1. Scheme of the experimental setup showing the excitation, the confocal microscope and both spectrometers. CoSY: Compact Saturation Spectroscopy, PD: Photodiode, FPI: Fabry-Pérot, FC: fiber coupler, CL/SL: cylindrical spherical lens

\footnotetext{
* Corresponding author: jan.rix@mailbox.tu-dresden.de
} 

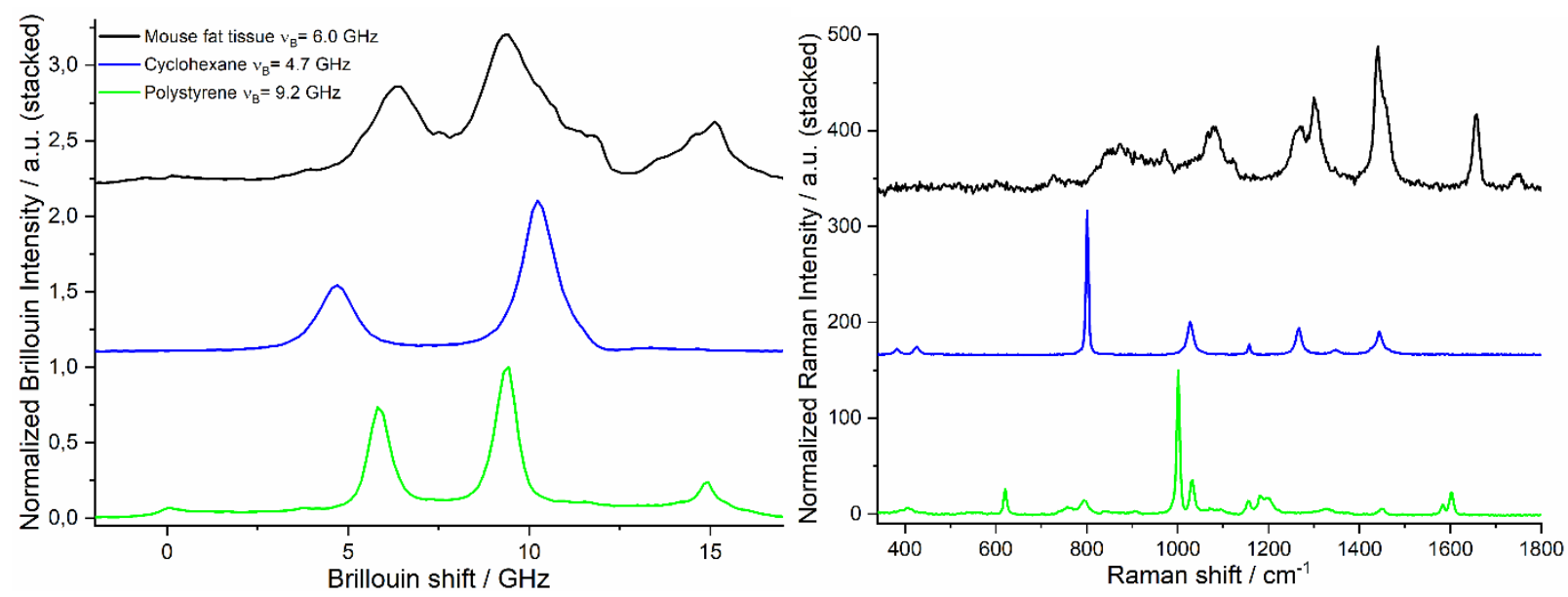

Fig. 2. Exemplary Brillouin and Raman spectra of polystyrene, cyclohexane and fat tissue of a mouse. Water has been used for axis calibration of the Brillouin spectra. As the free spectral range (FSR) of the VIPAs amounts $15 \mathrm{GHz}$, the Rayleigh bands appear at 0 $\mathrm{GHz}$ and $15 \mathrm{GHz}$, because of an incomplete filtering out of the elastically scattered light.

\section{Results}

A schematic representation of the system is given in Fig. 1. Scattering was excited using a laser source emitting at a wavelength of $780.24 \mathrm{~nm}$ that is best for opaque biological samples. The scattering radiation is acquired by a commercial confocal microscope (WITec Alpha 300R, WITec GmbH, Ulm, Germany). The Rayleigh and the Brillouin scattered light is separated from the Raman scattered light by a dichroic beam splitter (edge at 785 $\mathrm{nm}$ ) and transferred to the respective spectrometer by single mode fibers. For Raman spectroscopy, a standard Raman spectrometer (WITec UHTS 400, WITec GmbH, Ulm, Germany) has been used. A Brillouin spectrometer based on a two-stage virtually imaged phased array (VIPA) configuration, as first described by Scarcelli et al. [2], has been designed and assembled. The system consists entirely of commercial components and is adapted from a set-up by Schlüßler et al. [3]. Three combined spectra, which have been acquired simultaneously, are exemplary presented in Fig. 2. Whereas the Brillouin spectra indicate a substantial difference in the samples' biomechanics, the Raman spectra show their characteristic chemical fingerprints.

\section{Conclusion}

It was shown that first combined measurements on biological samples are possible with the described setup. In the near future the system will be dedicated to investigations of biomechanical properties of brain tumor cells, with the aim of better understanding progression, spreading and metastasis of aggressive tumors within the central nervous system.
We thank the NCT Dresden for financing the experimental system. Furthermore, Jan Rix gratefully acknowledges the financial support of the Free State of Saxony. Special thanks go to Dr.-Ing. Raimund Schlüßler for the precious help and the fruitful discussions.

\section{References}

1. M. Troyanova, Z. Meng, V.V. Yakovlev, Biomed. Opt. Express 10, pp. 1774-1781 (2019)

2. G. Scarcelli, P. Kim, S. H. Yun, Opt. Lett. 33, pp. 2979-2981 (2008)

3. R. Schlüßler, S. Möllmert, S. Abuhattum, G. Cojoc, P. Müller, K. Kim, C. Möckel, C. Zimmermann, J. Czarske, J. Guck, Biophys. J. 115, pp. 911-923 (2018) 